To see whether that decomposition was not due to pyrolysis, we submitted pure cyclohexyl sulphite to distillation under atmospheric pressure. Decomposition took place, with an abundant evolution of sulphur dioxide; rectification of the distillate showed pure dicyclohexyl oxide, b.p. $270^{\circ} / 760 \mathrm{~mm}$. and $131^{\circ} / 12 \mathrm{~mm}$.

Hence the following modification of Darzens' procedure : 1 mole of thionyl chloride is introduced into 2 moles of hydroxy compound, with circulation of a stream of dry air, until hydrogen chloride ceases to be evolved; $\mathrm{I} \cdot 2$ more moles of thionyl chloride is then added and left for 24 hours. Only then some drops of pyridine are added, and the mixture is heated for four hours on a water-bath to remove the sulphur dioxide. Pyridine only lowers the decomposition point of the corresponding chlorosulphite. Distillation then gives an excellent yield of cyclohexyl chloride (b.p., $141^{\circ} \mathrm{C}$.; $n_{D}, 1.462 ; D^{18}, 0.9765$ ).

The same procedure gave us, with yields of nearly 95 per cent, ethyl $\alpha$-chloropropionate, ethyl phenylchloroacetate, ethyl diphenylchloroacetate.

Établissements Théraplix,

98 rue de Sèvres, Paris VITe. Aug. 6.

${ }^{2}$ Human and Mills, Nature, 158, 876 (1946).

3 Carré and Libermann, C.R. Acau. Sci. Paris, 199, 1422 (1934).

- Gerrard and French, Nature, 159, 263 (1947).

- Darzens, C.R. Acad. Sci. Paris, 152, 1314 (1912).

- Libermann, Ion, 4, 40-41, 813 (Madrid, 1944).

- Kyrides, J. Amer. Chem. Soc., 66, 1006 (1944).

- Libermann, XIth Congress of Pure and Applied Chemistry, London, 1947 (in the press).

\section{Response of New-born Children to Hyper- tonic Solutions of Sodium Chloride and of Urea}

From all that is known about the renal function of new-born infants, it is now clear that their glomerular filtration-rates are considerably lower than those of adults, and also their clearances of urea and sodium chloride ${ }^{1}$. These discoveries have an important bearing on clinical practice, but much more information is required about the way in which the kidney of an infant reacts to changes in the internal environment. Accordingly, some experiments have been made to study the response of the kidney in new-born infants to a considerable rise in the osmotic pressure of the body. The children used for these experiments had all been born with inoperable meningo-myeloceles. Healthy adults have been used as controls.

All the subjects were deprived of water for about 12 hours before the tests began. Sufficient sodium chloride was then given intravenously as a 10 per cent solution to raise the serum concentration of chlorine to about $135 \mathrm{~m} . e q . / \mathrm{l}$. in the adults and to about $147 \mathrm{~m} . e q . / 1$. in the infants. The results may be summarized as follows :

(1) A diuresis was produced in both infants and adults.

(2) On a basis of surface area, this diuresis was much smaller in infants than in adults. The greatest rate of flow in an infant has so far been $2 \cdot 7$ c.c. $/ 1 \cdot 73$ sq. m./min. All adults have exceeded a rate of 7.6 c.c. 1.73 sq. m./min., and one has reached a value of $19 \cdot 1$ e.c. $/ 1.73 \mathrm{sq}$. $\mathrm{m} . / \mathrm{min}$.

(3) The administration of sodium chloride tended to raise rather than lower the osmotic pressure of the urine in infancy, but it greatly reduced the osmotic pressure of the urine in adults ${ }^{2}$.

(4) The infants excreted the administered sodium chloride much more slowly than adults (see graph). In one hour they excreted less than 1 per cont of the dose given, and in four hours 9.5 per cent. In the same times adults excreted 11 and 40 per cent of the dose. When comparisons were made on the basis of surface area, it was found that the maximum rate of excretion of chloride by adults was eight times that of infants. A feature of the experiments in both adults and infants has been that the diuresis produced by the salt solutions fell off while the concentration in the plasma was still high. No satisfactory explanation of this can be suggested at present.

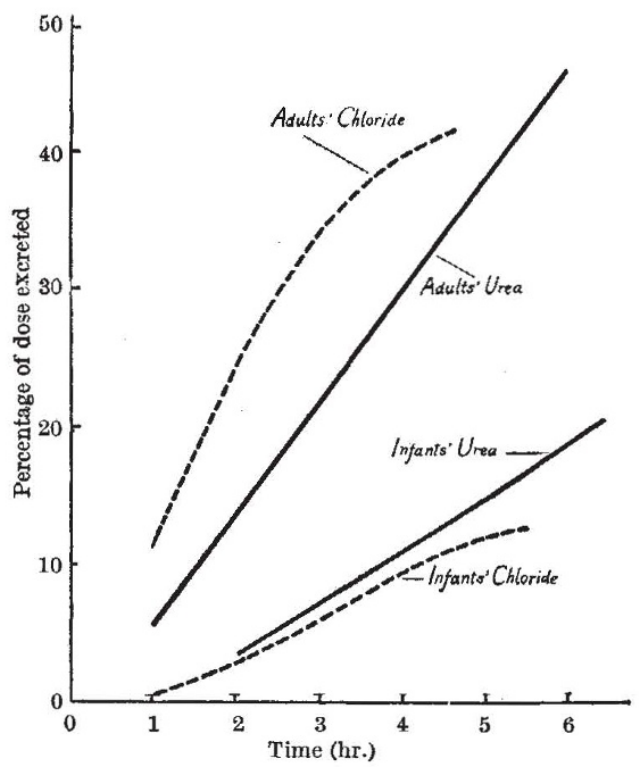

Urea has been given by mouth as a concentrated solution to adults and infants in quantities sufficient to raise the blood urea to about $30 \mathrm{~m} . \mathrm{mol}$./l. The response at both ages has had the same general charæcters as the response to sodium chloride (see graph), but the differences between the adults and the infants have not been so great and the maximum output of urea by the adults per unit of surface area has not been more than six times as great as that of the infants. The diuresis, moreover, which at its peak was only about one third as great as that produced by the salt solutions, declined more slowly and the rate of excretion of the urea was better maintained.

It is evident that the kidneys of human infants respond better than those of new-born rats to the administration of strong solutions of sodium chloride and urea ${ }^{3}$, but that the responses are essentially similar in nature. It is likely that these signs of immaturity will be demonstrable in all young mammals, but that no two species will show them to the same degree.

Department of Experimental Medicine,

$$
\text { R. F. A. Dean }
$$$$
\text { R. A. McCANCE }
$$

Cambridge.

Oct. 18.

${ }^{1}$ MeCance, R. A., and Young, W. F., J. Physiol., 99, 265 (1941).

${ }^{2}$ Hervey, G. R., MeCance, R. A., and Taylor, R. G. O., Nature, 157, 338 (1946)

s MeCance, R. A., and Wilkinson, E., J. Ph iol., 106, 256 (1947). 